\title{
Mecanismos alternativos de solución de conflictos. Análisis bibliométrico 2009-2018: base de datos Scopus
}

\author{
Alternative Dispute Resolution Mechanisms. 2009-2018 Bibliometric Analysis: \\ Scopus Database
}

Mecanismos alternativos para resolução de conflitos. Análise bibliométrica 2009-2018: banco de dados Scopus

Blanca Noemí Silva Gutiérrez

Universidad de Guadalajara, Centro Universitario de Ciencias Económico Administrativas,

México

bsilva@cucea.udg.mx https://orcid.org/0000-0001-8851-7079

Jorge Alan Chávez Meléndez

Universidad de Guadalajara, Centro Universitario de Ciencias Económico Administrativas,

México

alan.chavez.melendez@gmail.com https://orcid.org/0000-0002-5131-9914

\section{Resumen}

El objetivo del presente estudio consistió en realizar un análisis bibliométrico de la producción académica en el campo de los mecanismos alternativos de solución de conflictos (MASC) en la base de datos Scopus. Lo anterior con el propósito de identificar en esta temática los indicadores por año, autor, afiliación, país o territorio, tipo de documento y área del conocimiento. Se aplicó la metodología de análisis bibliométrico descrita por Escorcia (2008) y Arias (28 de noviembre de 2014), que consiste en realizar técnicas estadísticas que tienen como propósito evaluar el proceso de investigación y su evolución. El estudio fue de carácter cuantitativo longitudinal con descripción de tendencias de la producción científica. Como parte de los principales resultados, se identificó que el 2015 fue el año que contó con el mayor número de registros sobre el tema, con un total de 80 documentos publicados en la base de datos, 


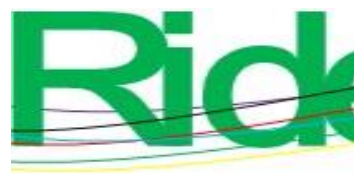

Revista lberoamericana para la

Investigación y el Desarrollo Educativo

ISSN 2007 - 7467

considerando que Scopus cuenta con registros desde el año de 1985. Mientras que el autor que más publicaciones tuvo sobre el término MASC fue el investigador Cheung So, de la Universidad de Hong Kong, con un total de 19 documentos. De igual forma, se localizó que el país con más publicaciones en dicha base de datos fue Estados Unidos con 369 documentos entre los años de 1985 y 2018. Si bien en Scopus se tiene registro desde 1985, el periodo de análisis correspondió al decenio del 2009 al 2018. A pesar de que se observó un incremento en las publicaciones sobre los MASC, cabe destacar que se identificó un decremento significativo en la publicación de documentos alusivos al tema a partir del año 2015.

Palabras clave: análisis bibliométrico, conflicto, justicia alternativa, métodos alternos, Scopus.

\section{Abstract}

The objective of this study was to perform a bibliometric analysis of academic production in the field of alternative dispute resolution (ADR) mechanisms in the Scopus database. The above with the purpose of identifying in this subject the indicators by year, author, affiliation, country or territory, type of document and knowledge area. The methodology of bibliometric analysis described by Escorcia (2008) and Arias (November 28, 2014) was applied, which consists in performing statistical techniques that have the purpose of evaluating the research process and its evolution. The study was of a quantitative longitudinal nature with a description of trends in scientific production. As part of the main results, it was identified that 2015 was the year that had the highest production record on the subject, with a total of 80 documents published in the database, considering that Scopus has records since 1985. While the author who had more publications on the term $A D R$ was Cheung So, researcher at the University of Hong Kong, with a total of 19 documents. Likewise, it was found that the country that had the most publications in said database was the United States of America with 369 documents between 1985 and 2018. Although Scopus has a record since 1985, the period of analysis corresponded to the decade from 2009 to 2018. Although there was an increase in the publications on the ADR mechanisms, it should be noted that a significant decrease was identified in the publication of documents alluding to the subject as of 2015.

Keywords: bibliometric analysis, conflict, alternative justice, alternative methods, Scopus. 


\section{Resumo}

O objetivo do presente estudo foi realizar uma análise bibliométrica da produção acadêmica no campo dos mecanismos alternativos de resolução de conflitos (MASC) na base de dados Scopus. O exposto a fim de identificar os indicadores por ano, autor, afiliação, país ou território, tipo de documento e área de conhecimento sobre este tópico. Foi aplicada a metodologia de análise bibliométrica descrita por Escorcia (2008) e Arias (28 de novembro de 2014), que consiste na execução de técnicas estatísticas cujo objetivo é avaliar o processo de pesquisa e sua evolução. O estudo foi de natureza quantitativa longitudinal, com uma descrição das tendências da produção científica. Como parte dos principais resultados, identificou-se que 2015 foi o ano com o maior número de registros sobre o assunto, com um total de 80 documentos publicados no banco de dados, considerando que o Scopus possui registros desde o ano de 1985. Enquanto o autor com mais publicações sobre o termo MASC foi o pesquisador Cheung So, da Universidade de Hong Kong, com um total de 19 documentos. Da mesma forma, verificou-se que o país com maior número de publicações no referido banco de dados eram os Estados Unidos com 369 documentos entre 1985 e 2018. Embora o Scopus tenha um histórico desde 1985, o período de análise correspondeu à década de 2009 até 2018. Embora tenha sido observado um aumento nas publicações do MASC, deve-se notar que houve uma redução significativa na publicação de documentos alusivos ao assunto a partir do ano de 2015.

Palavras-chave: análise bibliométrica, conflito, justiça alternativa, métodos alternativos, Scopus.

Fecha Recepción: Octubre 2019

Fecha Aceptación: Marzo 2020

\section{Introducción}

\section{Los mecanismos alternativos de solución de conflictos}

El acceso a la justicia se trata indudablemente de un derecho humano que garantiza a cualquier persona el medio a través del cual puede encontrar la forma de dar solución a sus dificultades cotidianas en caso de tener un conflicto (Castillo y Bautista, 2018; Cornelio, 2014). No obstante, desafortunadamente la realidad respecto a dicho acceso se encuentra en función de las aptitudes y circunstancias que tenga cada país para dar solución a los conflictos que se puedan presentar, así como de diversas situaciones que resultan propias de cada individuo, tales como el entorno socioeconómico. 


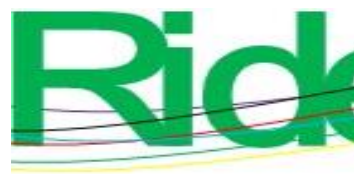

Revista Iberoamericana para la

Investigación y el Desarrollo Educativo ISSN 2007 - 7467

En la actualidad, la capacidad para solucionar conflictos vinculados al sistema de justicia en diversos países se ha visto rebasada debido a la excesiva demanda y la acumulación de controversias, por lo que, como resultado, se ha generado un atraso significativo en la administración de justicia. En este contexto, desde hace varios años surge en México la llamada justicia alternativa y, con ella, los mecanismos alternativos de solución de conflictos (MASC). La intención fundamental es lograr un cambio de paradigma en el abordaje de las controversias y la impartición de justicia, además de obtener un descongestionamiento de la saturación de trabajo del sistema de justicia tradicional.

El término justicia alternativa puede entenderse, de acuerdo con Mondragón (2007, citado en Nava y Breceda, 2017) y Estalvillo (2004), como una estructura procesal diferente a la jurisdiccional que busca la solución de controversias o conflictos entre particulares a través de los MASC, los cuales incluyen la mediación, conciliación, negociación y el arbitraje. Dichos métodos proporcionan ventajas como una participación más activa que involucra a las partes afectadas en el proceso de solución, la optimización del tiempo que tomaría el proceso legal mediante la vía tradicional y una de las más significativas: la ventaja económica que estos ofrecen. Lo anterior, siguiendo aquí a Cadenas (2017), tiene el objetivo de "permitir una mayor participación de los ciudadanos en la resolución de los conflictos, una reducción de las tensiones sociales y llegar así a una mejora de las relaciones sociales y de la calidad de vida” (p. 1631).

El origen de los métodos alternativos se ubica en Estados Unidos de América en el año de 1970; un poco más tarde se practicarían en Canadá y Australia. En los años 90 se institucionalizaron estos mecanismos cuando el Congreso de Estados Unidos impuso que todas las cortes federales llevaran a cabo la implementación y uso de los procedimientos de resolución de conflictos. En la actualidad, los países del continente europeo aplican estos métodos en la solución y resolución de conflictos. En España son 13 las comunidades autónomas que han venido practicando la mediación y negociación en los conflictos colectivos laborales (Cornelio, 2014).

Es importante señalar que, con el surgimiento de la justicia alternativa y los MASC, no se busca en ningún momento sustituir lo llevado a cabo por el sistema tradicional de justicia, sino fungir como una vía alterna que coadyuve a aliviar la carga de trabajo de este y que, de igual manera, la sociedad se vea beneficiada a través de métodos de resolución de conflictos que puedan resultar más eficientes según sea la problemática que se busque resolver. 


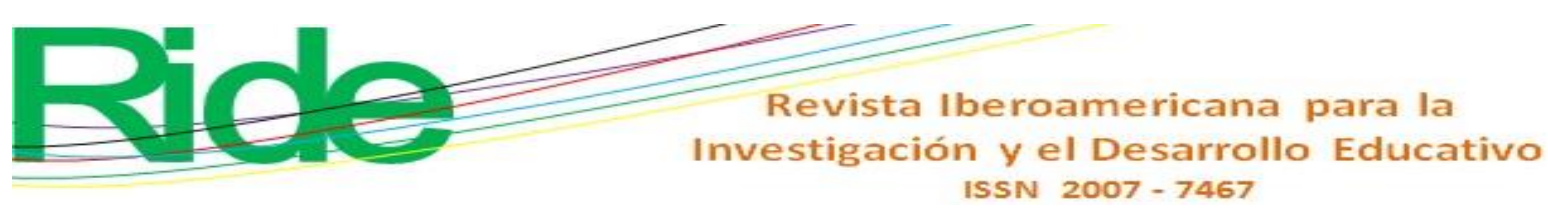

\section{El análisis bibliométrico}

El propósito esencial que tiene un trabajo de investigación es el de su divulgación. A sabiendas de lo anterior, los miembros de la comunidad científica buscan dar a conocer los avances en los que han trabajado en sus respectivas áreas del conocimiento, teniendo como resultado lo que comúnmente conocemos como producción científica.

Una técnica que resulta eficaz para conocer y medir cuánta producción científica existe en determinado campo disciplinar es el análisis bibliométrico. Y no solo eso, también es una herramienta importante para la organización de colecciones bibliográficas.

El análisis bibliométrico presenta generalmente de forma visual y ordenada el comportamiento que ha tenido la producción científica en cualquier área de investigación. Gracias a los diversos indicadores que esta puede presentar, es posible conocer datos de gran relevancia sobre el tema que se esté estudiando. Como resultado se obtiene un panorama general no solo del estado de la cuestión como tal, sino también de su evolución.

De acuerdo con Velázquez, Robledillo, Mangas, Veiga y Maqueda (2013), la primera referencia del concepto de bibliometría fue hecha por Paul Otlet en el año de 1934. En su trabajo, Otlet resalta la necesidad e importancia de esta ciencia de medición y describe los primeros indicadores que hacen posible la cuantificación de la producción, así como de su organización.

Miguel y Dimitri (2013), por su parte, dentro de los antecedentes más antiguos acerca de estudios o análisis bibliométricos que han sido localizados en la literatura, citan los trabajos de Cole y Eales de 1917, quienes realizaron publicaciones de anatomía comparada del periodo 1550-1860, así como el de Hulme de 1923, bibliotecario de la British Patent Office, que llevó a cabo un análisis estadístico sobre publicaciones de historia de la ciencia, y el trabajo de Gross y Gross en 1927 sobre las referencias incluidas en artículos de revistas de química indizadas en el Journal of the American Chemical Society. También Miguel y Dimitri (2013) destacan los estudios que dieron lugar a la formulación de las "leyes bibliométricas", tales como la ley de productividad científica de Lotka (1926), la ley de dispersión de la literatura sobre temas específicos de Bradford (1934), la ley de Zipf (1935) sobre la distribución de las palabras en un texto y las leyes de crecimiento exponencial de la ciencia y de obsolescencia de la literatura científica de Price (1963).

Al respecto, Castillo (2002) establece la siguiente clasificación de las leyes bibliométricas (ver tabla 1): 


\begin{tabular}{|c|c|}
\hline \multicolumn{2}{|c|}{ Tabla 1. Clasificación de las leyes bibliométricas } \\
\hline $\begin{array}{c}\text { Leyes que inciden en la literatura } \\
\text { científica }\end{array}$ & $\begin{array}{l}\text { Leyes que inciden en los autores y su } \\
\text { producción }\end{array}$ \\
\hline Ley de crecimiento exponencial & Ley de productividad \\
\hline $\begin{array}{l}\text { Ley de obsolescencia o } \\
\text { envejecimiento }\end{array}$ & Ley de impacto o visibilidad \\
\hline Ley de dispersión & \\
\hline
\end{tabular}

Fuente: Elaboración propia con base en Castillo (2002)

En 1969, Alan Pritchard crea su propia definición de bibliometría: "La aplicación de los métodos estadísticos y matemáticos dispuestos para definir los procesos de la comunicación escrita y la naturaleza y el desarrollo de las disciplinas científicas mediante técnicas de cuento y análisis de dicha comunicación” (Velázquez et al., 2013, p. 385). También se utilizan frecuentemente otros términos como cienciometría, informetría, bibliotecometría, webmetría; cada uno de ellos hace referencia a estudios métricos aplicados a fenómenos u objetos específicos, aunque en ocasiones suelen ser utilizados como sinónimos del término bibliometría (Sengupta, 1992).

Existen otras definiciones más recientes que exponen el significado de la bibliometría. Por ejemplo, la propuesta por Pérez et al. (2003, citados en Herrera, Núñez, Tobón y Arias (2009):

Un compilado de técnicas y procedimientos para estudiar la naturaleza y comportamiento de una disciplina mediante la sistematización y análisis de los diversos aspectos de la comunicación escrita, teniendo en cuenta una metodología específica que se ocupa del análisis cuantitativo de determinadas variables presentes en un texto publicado (p. 63).

Por otro lado, para Camps (2009) la bibliometría trata de lo puesto a continuación:

La ciencia que permite el análisis cuantitativo de la producción científica a través de la literatura, estudiando la naturaleza y el curso de una disciplina científica. Los indicadores bibliométricos se utilizan ampliamente para la evaluación de investigadores, instituciones y países, pero los datos que nos arrojan no deben interpretarse de manera aislada, como se hace frecuentemente con el factor impacto (p. 5).

Para Aström (2007), hace referencia a un campo de investigación emergente, cuya genealogía recorre las ramas de la bibliotecología y la ciencia de la información, que ha logrado un gran desarrollo en las últimas décadas. 


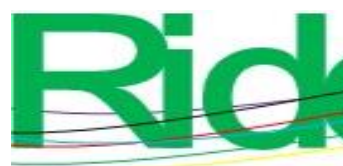

Revista lberoamericana para la

Investigación y el Desarrollo Educativo ISSN $2007-7467$

Mientras que para Escorcia (2008) la bibliometría se define como "una subdisciplina de la cienciometría que proporciona información sobre los resultados del proceso de investigación, su volumen, evolución, visibilidad y estructura. Así permiten valorar la actividad científica y el impacto tanto de la investigación como de las fuentes" (p. 13).

Para medir y evaluar diversos aspectos de la investigación científica, la bibliometría ha generado un sistema de indicadores bibliométricos, a saber:

- Los temas tratados

- $\quad$ El impacto o visibilidad

- $\quad$ Tipo de documentos consultados

- $\quad$ Actualidad y vigencia de las referencias citadas

- $\quad$ Dispersión de las publicaciones

- $\quad$ Los idiomas de publicación

- $\quad$ El origen de la bibliografía citada (nacional y extranjera) (Arias, 2017 pp. 81-82)

La aplicación de este tipo de análisis se encuentra fundamentada en mostrar la evolución que ha tenido la ciencia con base en determinada información y datos cuantitativos, así como para conocer las tendencias que presentan dicha evolución. Por lo tanto, y en este sentido, la presente investigación tuvo como objetivo principal conocer y describir la actividad científica y producción de información con respecto a los MASC registrada en la base de datos Scopus entre 2009 y 2018, y así obtener información acerca de las publicaciones que existen en la citada plataforma sobre el tema en dicho periodo, así como los autores que más han publicado al respecto, los países que más han contribuido con el tópico, entre otros.

\section{Método}

Se aplicó la metodología para los estudios bibliométricos descrita por Escorcia (2008) y Arias (28 de noviembre de 2014), que consiste en aplicar técnicas estadísticas que tienen como propósito evaluar el proceso de investigación y la evolución de una temática determinada, en este caso, en lo relativo a la producción académica en el campo de MASC. El estudio fue de carácter cuantitativo longitudinal y comprende la descripción de las tendencias de la producción científica del periodo que va de 2009 a diciembre de 2018. Lo anterior con base en la detección del incremento significativo en la producción de dicho periodo. No se incluyó el año 2019 debido a que, al inicio de este estudio, no se contaba con los datos del año completo. 


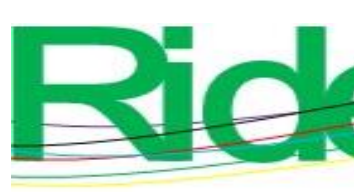

Revista Iberoamericana para la

Investigación y el Desarrollo Educativo

ISSN 2007 - 7467

Como parte del análisis de tipo descriptivo, se realizó una búsqueda de documentos sobre los MASC. Al tratarse de una de base de datos cuyos resultados y publicaciones son en idioma inglés, y por tratarse de un análisis que se plantea en términos internacionales, se llevó a cabo la traducción de dicho concepto. De esta manera, se identificaron las palabras clave más recurrentes en Scopus con la intención de delimitar la búsqueda específica y se encontró alternative dispute resolution (ADR) como la cadena más representativa entre las publicaciones internacionales.

Posteriormente, se llevó a cabo la búsqueda de documentos con el término específico antes mencionado, tomando como criterio que los resultados arrojaran documentos pertinentes a través del título, el abstract y las palabras clave, para asegurar que el resultado fuera confiable. Asimismo, se refinaron las búsquedas aplicando los operadores booleanos, gracias a lo cual se logró una mayor exactitud, y se generó un análisis de los resultados obtenidos a partir de la base de datos. Con ello se obtuvo información histórica respecto a la cantidad de documentos publicados por año, autor, afiliación de los autores, país, tipo de documento y área del conocimiento.

Con respecto a la elección de la base de datos para llevar a cabo el análisis bibliométrico, inicialmente se realizó la búsqueda en dos de las más reconocidas a nivel internacional: Scopus y Web of Science; sin embargo, esta última arrojó únicamente nueve documentos sobre los MASC, por lo que dicho resultado se consideró insuficiente para cumplir el objetivo de la presente investigación. Por lo tanto, se tomó la decisión de enfocar el análisis exclusivamente en la base de datos Scopus, debido, además, a la relevancia y especialización a nivel internacional en su tipo. Actualmente, la Universidad de Guadalajara cuenta con acceso al repositorio general de bases de datos especializadas y multidisciplinarias, entre estas últimas se encuentra Scopus, considerada la mayor base de datos de citas y resúmenes de bibliografía revisada por pares. Cabe mencionar que en Scopus se incluyen revistas científicas, libros y actas de conferencias de ramas como la ciencia y tecnología, ciencias sociales, artes y humanidades, entre otras.

\section{Resultados}

Como producción total sobre el término $A D R$ (recordemos que se trata del equivalente de MASC en inglés) la base de datos Scopus cuenta con un registro de 1132 documentos hasta el año 2018. Si bien el primer registro data de 1985, para efectos del presente estudio se tomó como delimitación temporal el periodo 2009-2018, como ya se señaló anteriormente. A continuación se presentan los datos completos acerca de la producción científica en la citada base de datos con la intención de ofrecer un panorama sobre su evolución desde el año en que 


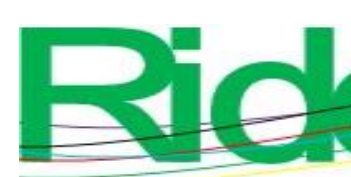

Revista Iberoamericana para la Investigación y el Desarrollo Educativo ISSN $2007-7467$

\section{Producción científica por año}

Durante el periodo objeto del presente estudio (2009-2018), la base de datos registró un total de 1132 documentos publicados sobre MASC. Como ya se mencionó, el año 2015 fue en el que se encontró una mayor producción, con 80 documentos, mientras que, por el lado contrario, el año en el que se tuvo el menor registro fue 1985, con solo dos documentos publicados. Este dato resulta evidente ya que se trata del primer año en el que se tiene registro, mientras que en el año posterior inmediato (1986) no se tienen registros de algún documento publicado, y es a partir del siguiente que la producción fue incrementando, con siete documentos registrados en 1987. A partir de dicha fecha, la producción fue incrementando, manteniéndose una media de 22.80 documentos por año hasta llegar al 2009. La figura 2 muestra la evolución de este indicador durante el periodo de producción de 2009-2018.

Figura 2. Evolución en la publicación de documentos sobre MASC durante el periodo 20092018 en Scopus

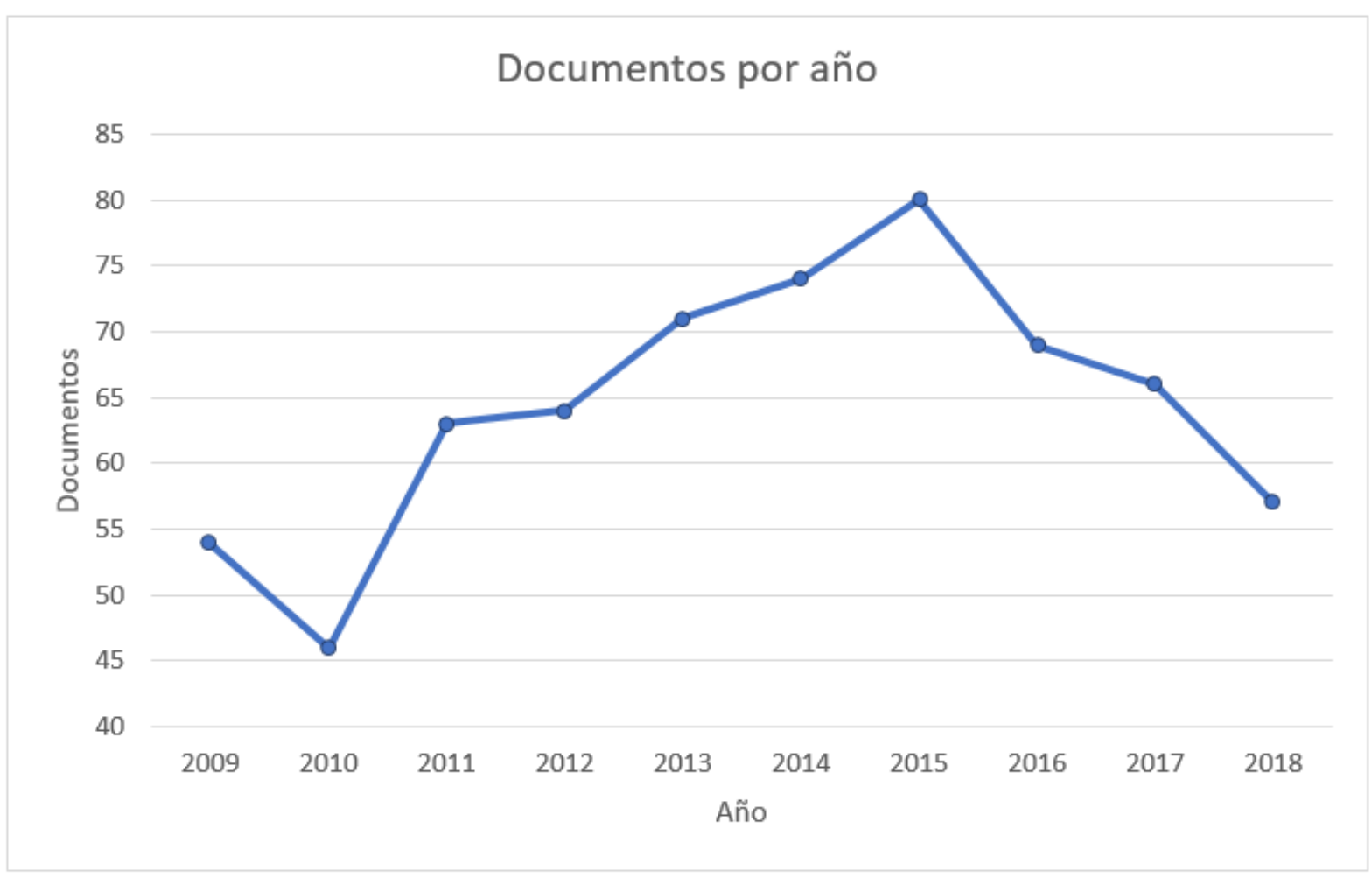

Fuente: Elaboración propia con base en Scopus 


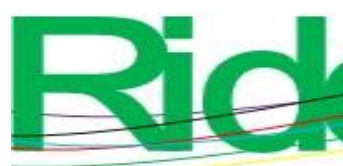

Revista lberoamericana para la Investigación y el Desarrollo Educativo ISSN $2007-7467$

\section{Producción científica por autor}

En lo que respecta a esta categoría, se encontró que el autor que más publicaciones realizó durante el periodo de investigación fue S. Cheung, con un total de 19 documentos publicados, seguido de J. Zeleznikow con 10 documentos. Mientras que son 114 los autores que representan la minoría de registros publicados con un total de dos publicaciones cada uno desde 1985 hasta el 2018.

Figura 3. Evolución en la publicación de documentos sobre MASC durante el periodo 2009-

2018 por autor en la base de datos Scopus

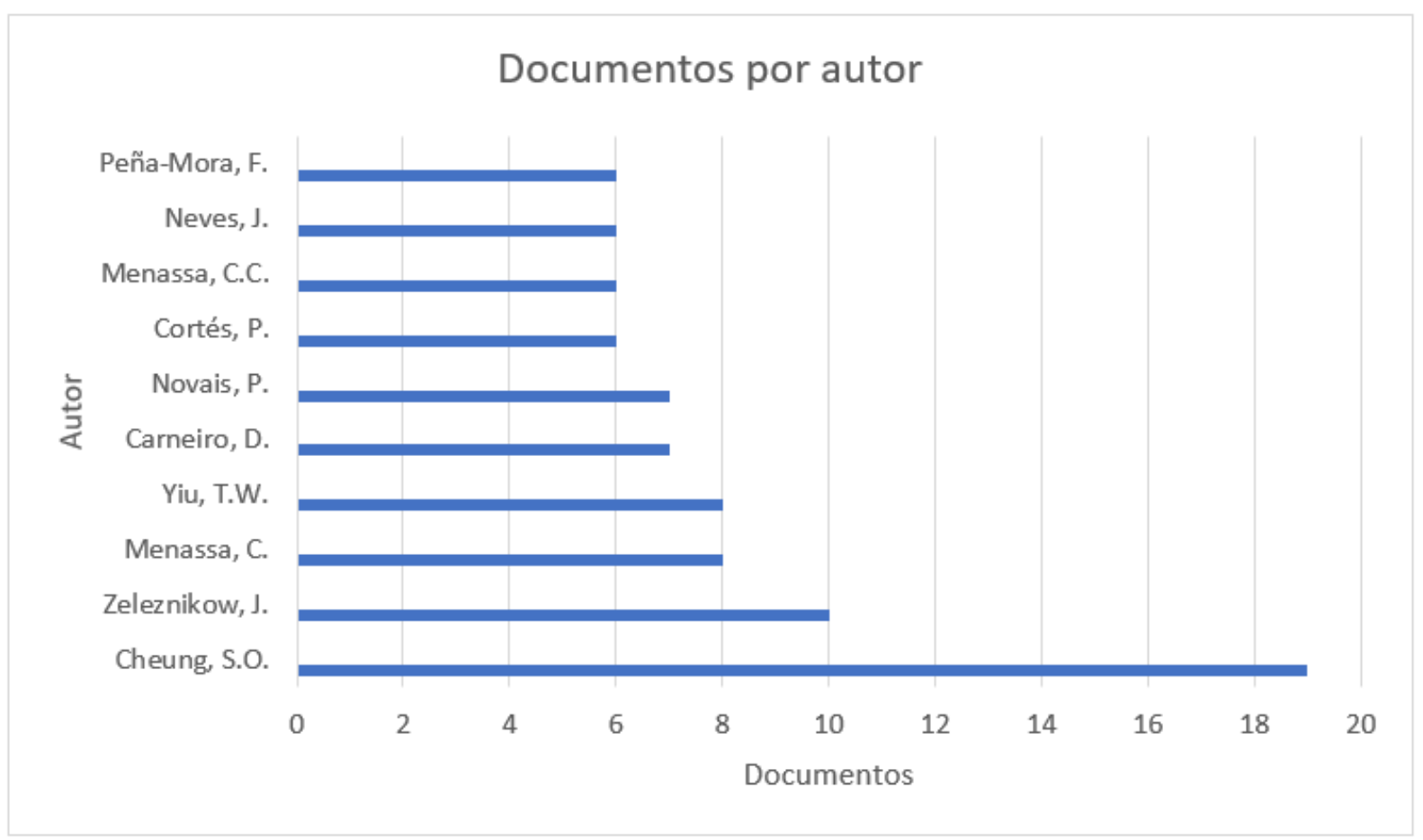

Fuente: Elaboración propia con base en Scopus

\section{Producción científica por afiliación de los autores}

De acuerdo con la información localizada en la base de datos Scopus, se identificó que, de los documentos que se publicaron durante el periodo analizado, la afiliación de sus autores conforma un muy variado mapa de instituciones educativas, entre las que destacan la Universidad de Hong Kong, con 23 documentos publicados por autores afiliados a esta, la mayor cantidad en el rubro, seguida de la Universidad de Oxford con un total de 12 documentos y la Universidad Victoria de Melbourne con una representación total de 11 documentos . Demuestran una menor representación 61 universidades o instituciones con solamente dos publicaciones cada una: el Instituto de Manejo de Conflictos Médicos, la Universidad de 


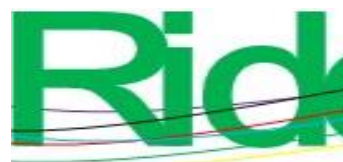

Revista Iberoamericana para la Investigación y el Desarrollo Educativo ISSN $2007-7467$

Westminster en Londres, la Universidad de Nevada en Estados Unidos o la Universidad de Dundee en Escocia, por mencionar tan solo algunas.

Figura 4. Distribución de documentos sobre MASC según la afiliación institucional de sus autores durante el periodo de 2009-2018 en la base de datos Scopus

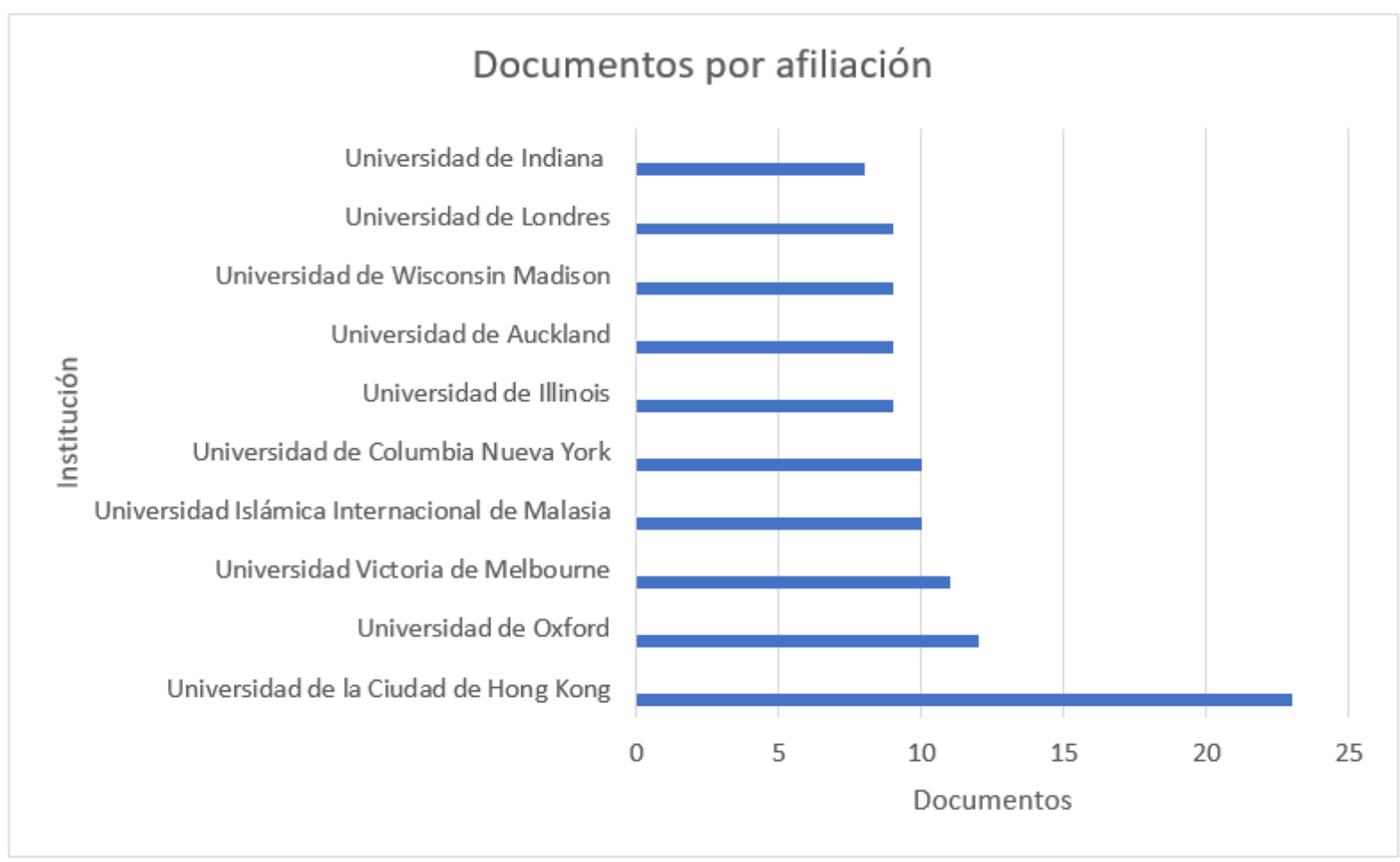

Elaboración propia con base en Scopus

\section{Producción científica por país o territorio}

Existen diversos países o territorios que contribuyen a la producción de investigaciones sobre el término MASC. A pesar de que pareciera que los países europeos tienen mayor aporte sobre el tema, la realidad es que el país que más contribuyó a la producción durante el periodo de análisis fue Estados Unidos de América, con un total de 369 documentos publicados en la citada base de datos, seguido por el Reino Unido con un total de 143 documentos, mientras que en tercer lugar se ubica Australia con 69 documentos. Como dato relevante destaca que México se ubica en el lugar 39 con un total de cuatro documentos. Por otro lado, los países que tienen menor producción científica son los Emiratos Árabes Unidos, Uganda, Trinidad y Tobago, Tanzania, Serbia, Arabia Saudita, Puerto Rico, Kenia, Irán, Egipto, Argentina, Barbados, entre otros. En el siguiente gráfico se muestran los primeros 10 países de producción científica respecto al tema aquí protagonista. 


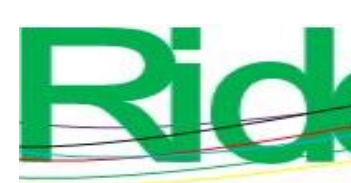

Revista Iberoamericana para la Investigación y el Desarrollo Educativo ISSN $2007-7467$

Figura 5. Países que más documentos publicaron sobre MASC en Scopus

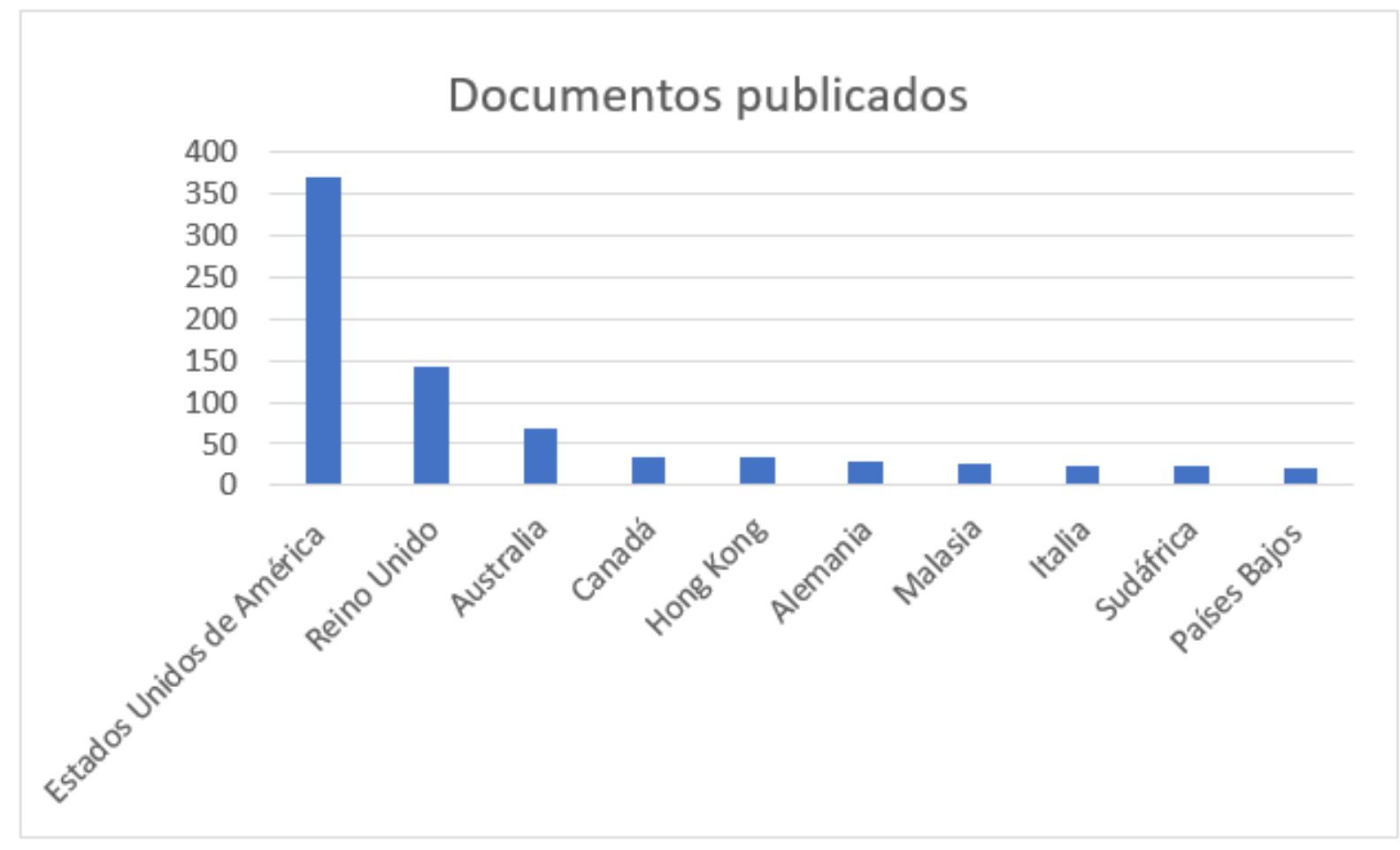

Fuente: Elaboración propia con base en Scopus

\section{Producción científica por tipo de documento}

Cuando se trata de divulgar el conocimiento, son distintas los formatos a los que un investigador puede recurrir, ya sea a través de artículos publicados en revistas indizadas, libros, capítulos de libros o ponencias. En lo que concierne a la publicación de documentos sobre el tema de MASC en la base de datos Scopus, la mayoría de estas publicaciones son realizadas a través de artículos científicos, un total de 682 artículos durante el periodo analizado, seguido de ponencias con un total de 144 documentos existentes; en tercer lugar se encuentran los capítulos de libro con 109 registros. A continuación, se muestra un gráfico (figura 6) con la información completa en lo que a publicación por tipo de documento respecta. 


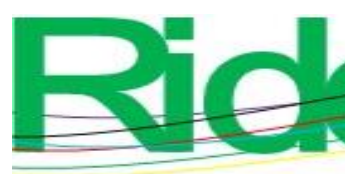

Revista lberoamericana para la Investigación y el Desarrollo Educativo ISSN $2007-7467$

Figura 6. Producción científica sobre MASC por tipo de documento

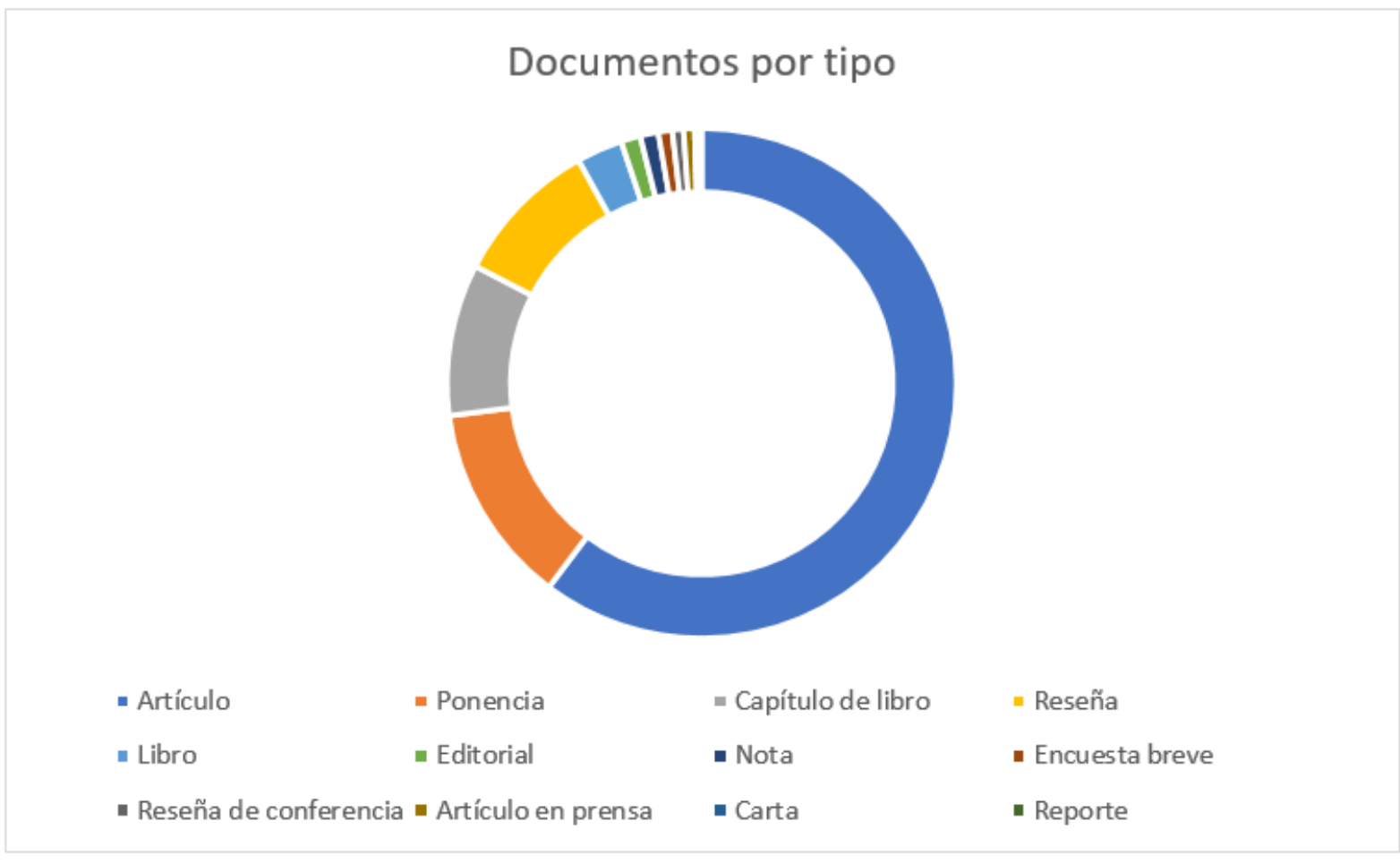

Fuente: Elaboración propia con base en Scopus

\section{Producción científica por área del conocimiento}

A pesar de que la producción científica relativa al término $M A S C$ va encaminada principalmente a cuestiones jurídicas o sociales, son diversas las áreas del conocimiento a través de las cuales se difunde dicho tema, incluyendo ingenierías, economía, artes y humanidades, hasta medicina o ciencias ambientales. A continuación, se presenta información relativa a las 10 principales áreas del conocimiento sobre las que se han realizado publicaciones respecto a los MASC. Cabe destacar que los criterios para la clasificación de dichas áreas es determinada por la misma base de datos. Así pues, destacan en primer lugar las ciencias sociales, entre las que predominan el derecho y la sociología, además de incluir algunas otras como la educación, historia, ciencias políticas, etc., con un total de 607 documentos identificados en dicha área. En segundo lugar está el área de negocios, gestión y contabilidad, con un total de 237 documentos. En tercer lugar figura la ciencia de la ingeniería con 199 documentos publicados. Por el contrario, las áreas que presentan un menor índice de publicación sobre dicho tema y de acuerdo con su clasificación son la odontología o la física y astronomía con un documento publicado cada una, además de la ingeniería química, la veterinaria o la neurociencia con tres, cuatro y cuatro publicaciones, respectivamente. 


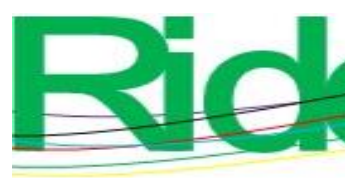

Revista Iberoamericana para la Investigación y el Desarrollo Educativo ISSN $2007-7467$

Figura 7. Producción científica sobre MASC por área del conocimiento

\section{Documentos por área del conocimiento}

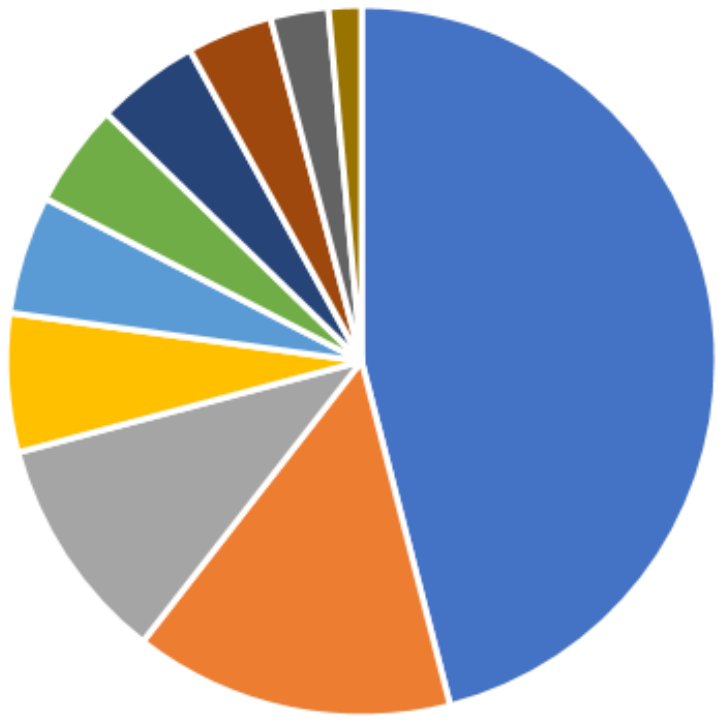

- Ciencias Sociales

- Gestión de Negocios y Contabilidad

- Ingenierías

- Economía, Econometría y Finanzas

- Artes y Humanidades

- Ciencias de la Computación

- Medicina

- Psicología

- Ciencias Ambientales

- Ciencias de la Decisión

Fuente: Elaboración propia con base en Scopus

\section{Discusión}

Durante el periodo evaluado, que comprendió de 1985 a 2018, se publicó un total de 1132 documentos relacionados con los MASC al interior de la base de datos Scopus, de los cuales una mayoría significativa eran artículos científicos, $60 \%$ aproximadamente, mientras que alrededor de $13 \%$ se trató de la publicación y presentación de ponencias, seguido por la publicación de capítulos de libro con un $9.5 \%$ aproximadamente, y en último lugar la publicación de libros sobre el citado tema, con una cantidad mínima (aproximadamente $3 \%$ ) en comparación con los artículos científicos.

Un dato destacable de la información antes señalada fue que la gran mayoría de los investigadores se están centrando en la publicación de artículos científicos como medio de divulgación para sus investigaciones, dejando de lado la producción de libros, ya que la base de datos no se limita a la publicación de artículos científicos, sino que esta también incluye la publicación de otro tipo de documentos: libros, capítulos de libro, etc.

Otro dato relevante fue el elevado número de documentos publicados en la base de datos que proceden de Estados Unidos y del continente europeo: en conjunto $60 \%$ de las publicaciones totales, con un aproximado de $30 \%$ cada uno, tanto Estados Unidos como el continente europeo. 


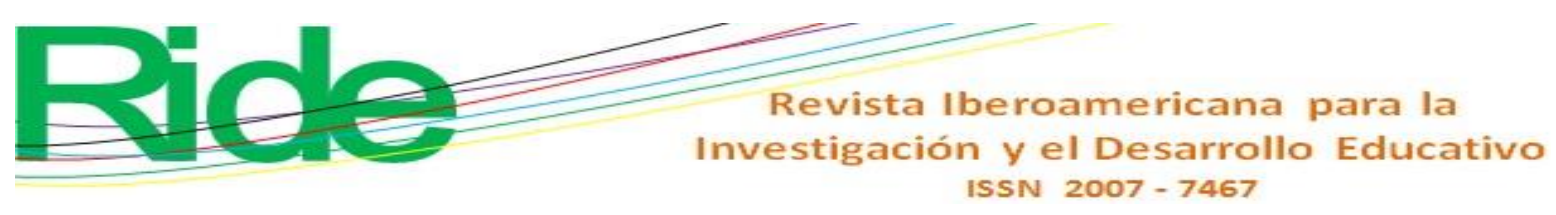

representa una clara necesidad de desarrollar investigaciones sobre el tema y generar a la vez la difusión del conocimiento en este campo.

De igual forma, al identificar los 10 países en donde se encuentran los organismos y universidades que mayor investigación han hecho y, por ende, mayor producción científica, la mayoría de ellos son europeos, quedando rezagados los países de habla hispana pertenecientes al continente americano.

El presente estudio cobra relevancia para el ámbito educativo al contribuir al escaso estado del conocimiento que actualmente existe en México sobre los MASC y su producción, así como también sienta las bases para que dichos métodos sean tomados en cuenta como herramientas que contribuyan al mejoramiento de las condiciones bajo las que se desempeñan tanto educadores como educandos.

Sirva como ejemplo la mediación escolar, cuyo propósito fundamental es el de mejorar la gestión de los conflictos que tienen su origen en el ámbito educativo, procurando establecer mejores relaciones interpersonales y promoviendo un ambiente que propicie el máximo aprovechamiento escolar.

Como ya se mencionó, esta investigación permitió conocer la tendencia en la producción del estudio de los MASC, sin embargo, una de sus limitaciones recae en haber realizado el análisis desde una misma base de datos, lo que podría dejar fuera investigaciones relevantes para el campo de estudio. Respecto a las fortalezas, se identifica la contribución que el presente análisis muestra para el estado del arte por ser una investigación pionera al respecto. Mientras que como áreas de debilidad y oportunidad se pueden señalar los pocos estudios científicos en idioma inglés que existen sobre los MASC, particularmente en Latinoamérica y México. 


\section{Referencias}

Arias, F. (28 de noviembre de 2014). Obsolescencia de la literatura científica. Ponencia presentada en la I Jornada del Centro de Investigación Edufisadred. Maracay, 28 de noviembre de 2014. Recuperado de http://www.docfoc.com/obsolescencia-de-laliteratura-cientifica-fidias-garias.

Arias, F. (2017). Obsolescencia de las referencias citadas: un mito académico persistente en la investigación universitaria venezolana. E-ciencias de la Información, 7(1), 78-90.

Aström, F. (2007). Changes in the LIS Research Front: Time-Sliced Cocitation Analyses of LIS Journal Articles, 1990-2004. Journal of the American Society for Information Science and Technology, 58(7), 947-957.

Cadenas, A. (2017). Mediación como justicia alternativa y diálogo social. San Cristóbal de La Laguna, España: Universidad de La Laguna.

Camps, D. (2009). El análisis bibliométrico de Universitas Scientiarum. Universitas Scientiarum, 14(1), 5-7.

Castillo, C. y Bautista, M. (2018). Acceso a la justicia alternativa: un reto complejo. Utopía y praxis latinoamericana, 23(2), 1-18.

Castillo, L. (2002). Introducción a la Bibliometría. Valencia, España: Universidad de Valencia.

Cornelio, E. (2014). Los mecanismos alternativos de solución de controversias como derecho humano. Barataria, (17), 81-95.

Escorcia, T. (2008). El análisis bibliométrico como herramienta para el seguimiento de publicaciones científicas, tesis y trabajos de grado. (tesis de licenciatura). Universidad Pontificia Javeriana de Colombia, Bogotá. Recuperado de http://www.javeriana.edu.co/biblos/tesis/ciencias/tesis209.pdf.

Estalvillo, F. (2004). Medios alternativos de solución de controversias. México: Porrúa.

Herrera, E., Núñez, A., Tobón, S. y Arias, D. (2009). Análisis bibliométrico de la depresión infantil. Pensamiento Psicológico, 5(12), 59-70.

Miguel, S. y Dimitri, P. (2013). La investigación en bibliometría en la Argentina: quiénes son y qué producen los autores argentinos que realizan estudios bibliométricos. Información, Cultura y Sociedad: Revista del Instituto de Investigaciones Bibliotecológicas, (29), 117-138.

Nava, W. y Breceda, J. (2017). Mecanismos Alternativos de Resolución de Conflictos: un acceso a la justicia consagrado como derecho humano en la Constitución Mexicana. Cuestiones Constitucionales, (37), 203-228.

Sengupta, I. (1992). Bibliometrics, Informetrics, Scientometrics and Librametrics: An Overview. Libri, 42(2), 75-98. 
Velázquez, D., Robledillo, A., Mangas, I., Veiga, J. y Maqueda, J. (2013). Análisis bibliométrico de la revista Medicina y Seguridad del Trabajo durante el periodo 20072012. Medicina y Seguridad del Trabajo, 59(233), 383-392.

\begin{tabular}{|c|c|}
\hline Rol de Contribución & Autor (es) \\
\hline Conceptualización & $\begin{array}{l}\text { Blanca Noemí Silva Gutiérrez - Principal } \\
\text { Jorge Alan Chávez Meléndez - Apoyo }\end{array}$ \\
\hline Metodología & $\begin{array}{l}\text { Blanca Noemí Silva Gutiérrez - Principal } \\
\text { Jorge Alan Chávez Meléndez - Igual }\end{array}$ \\
\hline Software & NO APLICA \\
\hline Validación & $\begin{array}{l}\text { Blanca Noemí Silva Gutiérrez - Igual } \\
\text { Jorge Alan Chávez Meléndez - Igual }\end{array}$ \\
\hline Análisis Formal & $\begin{array}{l}\text { Blanca Noemí Silva Gutiérrez - Igual } \\
\text { Jorge Alan Chávez Meléndez - Igual }\end{array}$ \\
\hline Investigación & $\begin{array}{l}\text { Blanca Noemí Silva Gutiérrez - Igual } \\
\text { Jorge Alan Chávez Meléndez - Igual }\end{array}$ \\
\hline Recursos & Blanca Noemí Silva Gutiérrez - Principal \\
\hline Curación de datos & $\begin{array}{l}\text { Blanca Noemí Silva Gutiérrez - Igual } \\
\text { Jorge Alan Chávez Meléndez - Igual }\end{array}$ \\
\hline $\begin{array}{l}\text { Escritura - Preparación del } \\
\text { borrador original }\end{array}$ & $\begin{array}{l}\text { Blanca Noemí Silva Gutiérrez - Igual } \\
\text { Jorge Alan Chávez Meléndez - Igual }\end{array}$ \\
\hline $\begin{array}{l}\text { Escritura - Revisión y } \\
\text { edición }\end{array}$ & $\begin{array}{l}\text { Blanca Noemí Silva Gutiérrez - Principal } \\
\text { Jorge Alan Chávez Meléndez - Apoyo }\end{array}$ \\
\hline Visualización & $\begin{array}{l}\text { Blanca Noemí Silva Gutiérrez - Igual } \\
\text { Jorge Alan Chávez Meléndez - Igual }\end{array}$ \\
\hline Supervisión & $\begin{array}{l}\text { Blanca Noemí Silva Gutiérrez - Principal } \\
\text { Jorge Alan Chávez Meléndez - Apoyo }\end{array}$ \\
\hline $\begin{array}{l}\text { Administración de } \\
\text { Proyectos }\end{array}$ & $\begin{array}{l}\text { Blanca Noemí Silva Gutiérrez - Principal } \\
\text { Jorge Alan Chávez Meléndez - Apoyo }\end{array}$ \\
\hline Adquisición de fondos & Blanca Noemí Silva Gutiérrez - Principal \\
\hline
\end{tabular}

